\title{
Safety and Efficacy of Nontuberculous Mycobacteria Treatment among Elderly Patients
}

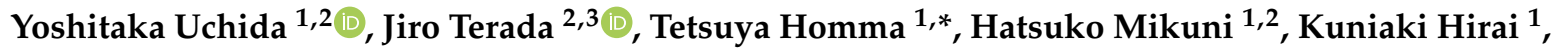 \\ Haruhisa Saito ${ }^{2}$, Ryoichi Honda ${ }^{2}$ and Hironori Sagara ${ }^{1}$ \\ 1 Department of Medicine, Division of Respiratory Medicine and Allergology, \\ Showa University School of Medicine, Tokyo 142-8555, Japan; for.u.goodlife@gmail.com (Y.U.); \\ ha.mikuni392@gmail.com (H.M.); medi123@infoseek.jp (K.H.); sagarah@med.showa-u.ac.jp (H.S.) \\ 2 Department of Respirology, Asahi General Hospital, Chiba 289-2511, Japan; jirotera@chiba-u.jp (J.T.); \\ hasaito@hospital.asahi.chiba.jp (H.S.); honda@sapmed.ac.jp (R.H.) \\ 3 Department of Respirology, Graduate School of Medicine, Chiba University, Chiba 260-8670, Japan \\ * Correspondence: oldham726@yahoo.co.jp; Tel.: +81-3-3784-8532
}

Received: 23 August 2020; Accepted: 28 September 2020; Published: 2 October 2020

\begin{abstract}
Background and objectives: Incidence rates of pulmonary nontuberculous mycobacterial (NTM) disease have been increasing, especially in the elderly population. Given the limited evidence regarding the safety and efficacy of NTM treatment, this study aimed to evaluate the same among elderly patients. Material and methods: Patients diagnosed with NTM disease at a tertiary hospital from January 2007 to December 2017 were enrolled and data were then retrospectively collected. Data of elderly patients who received antimycobacterial treatment were then analyzed. Results: A total of 161 patients satisfied the diagnostic criteria for NTM disease. There were 40 elderly patients who received treatments. Of the patients, $60 \%$ received the guideline oriented standard regimens. Single drug regimens were administered to $22.5 \%$ of patients. Only $55.0 \%$ of the patients were able to continue any treatment. Treatment-related discontinuation was observed in $44.4 \%$ of discontinued or changed patients. There were no significant differences in the characteristics of patients with or without adverse events. Patients who were able to continue the treatment for $>12$ months had a lower proportion of activities of daily living (ADL) disability (nine in 18, 50.0\% vs. three in 22, 13.6\%, $p=0.018$ ) and heart disease (six in $18,33.3 \%$ vs. $1 / 22,4.6 \%, p=0.033$ ). Sputum culture conversion was achieved in 28 out of $40(70.0 \%)$ elderly patients treated, and those who achieved sputum culture conversion had more standard regimens prescribed than those who failed sputum culture conversion (21 in 28, 75\% vs. 3 in 12, 25\%; $p=0.005$ ). Conclusion: Age may not be an obstacle for receiving the benefits of the treatment of NTM disease with a precise evaluation of patient's comorbidities. Furthermore, elderly patients without heart disease and ADL disability may have better rate of continuing the NTM treatment. The current study suggested that selecting standard regimens to treat pulmonary NTM is important for elderly patients.
\end{abstract}

Keywords: elderly; nontuberculous mycobacterium; NTM; treatment; safety

\section{Introduction}

The prevalence of pulmonary nontuberculous mycobacterial (NTM) disease has been increasing over the past few decades with varying rates in different parts of the world. Previous reports observed a sharp increase in the incidence rate of NTM disease in Japan (14.1 per 100,000 person-years in 2014, approximately 2.6 times higher than 2007) [1]. One study showed that the incidence and prevalence rates of NTM diseases in Japan were higher compared to other countries in North America, Europe, and Oceania [2]. Moreover, another study showed that among Japanese patients with NTM disease, 
$54.4 \%-86.6 \%$ were over 70 years old [3,4], which appeared to be much higher than in other countries [5]. Elderly patients are at risk for NTM-related mortality and disease progression [6,7]. In addition, elderly patients generally tend to have several and complicated comorbidities, which are considered one of the important predictors of death [8].

However, detailed information regarding elderly individuals with NTM disease is scarce. Previous large studies on NTM treatment cited by the guidelines [9] included elderly patients, but such patients' mean age was 49.5-75.5 [10-23]. Therefore, safety and efficacy of NTM disease treatment among elderly patients is still limited. This may lead to unrecommended treatment modifications, such as decreasing the number of antibiotics to single drug regimens $[6,9,24]$. Given the lack of evidence regarding the safety and efficacy of NTM treatment and the high prevalence of comorbidities in elderly patients, the present study aimed to evaluate the safety and efficacy of NTM treatment among elderly patients pulmonary NTM infection.

\section{Materials and Methods}

This retrospective study was conducted at Asahi General Hospital, a tertiary hospital in Japan. The study protocol was approved by the institutional ethics review committee (approval number 2018032023) and performed in accordance with the amended Declaration of Helsinki. Written informed consent was given and personal information was anonymized. Patients diagnosed with NTM disease were enrolled and analyzed.

The study included 181 patients whose age was above 20 years old with at least independent two positive cultures from expectorated sputum specimens or one positive culture from bronchial wash or bronchoalveolar lavage fluid specimens between January 2007 to December 2017. Patients with known HIV infection, cystic fibrosis, or who did not satisfy the following diagnostic criteria were excluded. The diagnosis was based on the 2007 American Thoracic Society criteria [25], which included the following: (1) symptoms of a pulmonary disease and exclusion of other lung diseases; (2) nodular or cavitary opacifications on chest radiograph or multifocal bronchiectasis with multiple small nodules on high-resolution computed tomography (HRCT) scan; and (3) at least two positive cultures from separately expectorated sputum specimens or one positive culture from bronchial wash or bronchoalveolar lavage (BAL) fluid. Among the 181 patients with positive culture, 161 met the diagnosis criteria. The patients who were below 70 years old was also excluded and age of 70 years old and above were analyzed. Although many other countries have defined elderly individuals as those aging $\geq 5$ years old, considering past study reporting many of Japanese patients with NTM disease are over 70 years old [3,4], we analyzed the patients who were 70 years old or more. We reviewed the clinical information, laboratory data including sputum culture, radiological findings, and used antibacterial regimens with $\mathrm{AE}$ information from 40 patients who were administrated the antibiotics to treat NTM disease.

Incidence of continuation of treatment, and adverse events (AEs) were determined from the medical records. Activities of daily living (ADL) disability was defined as the use of a wheelchair, any use of nursing care insurance, or any need for care with daily living recorded by any medical staff, including the care manager. The habit of alcohol was defined by at least three days or more per week of alcohol consumption with any amount. Respiratory diseases (asthma, chronic obstructive pulmonary disease (COPD), pulmonary fibrosis, etc.), malignant diseases, and heart diseases (heart failure, ischemic heart disease, arrythmia, etc.) were recorded to characterize patient's comorbidities. Chest radiological responses was assessed by chest radiograph or high-resolution computed tomography (HRCT), and assessment of chest radiograph and HRCT images were performed by at least one respiratory physician and one radiologist. Sputum culture conversion rates were also evaluated. When sputum specimens were not available, sputum culture conversion were marked as "improved". "Prescribed regimens" are defined as an antibiotic regimen that were used constantly. Data of changes of initial regimen to other regimen, treatment discontinuation, dosage adjustment, or regimen modification were collected. The duration of treatment was defined by the British Thoracic Society Guideline [9]. 
Patients who received at least 12 months of treatment were recorded as "continued", those who adjusted dosage or modified their regimens and were treated for at least 12 months were recorded as "changed", and those who were unable to continue their treatment for 12 months were recorded as "discontinued". Standard regimens were defined as combination of rifampicin (RFP), ethambutol (EB), and clarithromycin (CAM) for M. avium complex, combination of isoniazid (INH), RFP, and EB for M. kansasii, and combination of amikacin (AMK), CAM, and imipenem/cilastatin (IPM/CS) for M. abscessus.

Fisher's exact tests or Pearson's chi-squared statistic were used to compare group frequencies for categorical variables, while the Wilcoxon rank-sum test was used to compare group means for continuous variables. Significance was determined if P value was less than 0.05 . All analyses were performed using JMP ${ }^{\circledR}$ Pro 14.0.0 statistical software (SAS Institute Inc., Cary, NC, USA).

\section{Results}

\subsection{Study Population and Characteristics}

The baseline characteristics of treated elderly patients with NTM are described in Table 1. Mean age was 79.1 years old. Any comorbidities such as respiratory disease, malignancy, and heart disease were seen in $24 / 40(60 \%)$ patients. The most popular symptoms were cough $(\mathrm{n}=23,57.5 \%)$ and sputum $(n=20,50 \%)$. There were $9 / 40(22.5 \%)$ patients were prescribed only one drug and three out of $40(7.5 \%)$ patients were prescribed two drugs. Single drug regimens, which included erythromycin (EM), levofloxacin (LVFX), and CAM, were prescribed to five, one, and three elderly patients, respectively. Two drug regimens included EB + CAM, AMK + LVFX, and CAM + LVFX, each of which were prescribed to one elderly patient, respectively. Regimens with three or more drugs prescribed to elderly patients included RFP + EB + CAM $(n=22), I N H+R F P+E B(n=1), I P M / C S+$ Tobramycin + CAM $(n=1)$, Faropenem $($ FRPM $)+$ RFP + LVFX +CAM $(n=1), E B+C A M+\operatorname{LVFX}(n=1), C A M+F R P M+$ Sitafloxacin $(\mathrm{n}=1)$, AMK + CAM + IPM/CS $(\mathrm{n}=1)$.

Most patients were infected either with $M$. intracellulare $(\mathrm{n}=24)$, or M. avium $(\mathrm{n}=8)$, which accounted for $80 \%$ of patients (Figure 1). Other species included M. kansasii $(\mathrm{n}=3)$, M. abscessus $(\mathrm{n}=3)$, M. chelonae $(\mathrm{n}=1)$, and rapidly growing mycobacteria, which was not able to be determined $(\mathrm{n}=1)$.

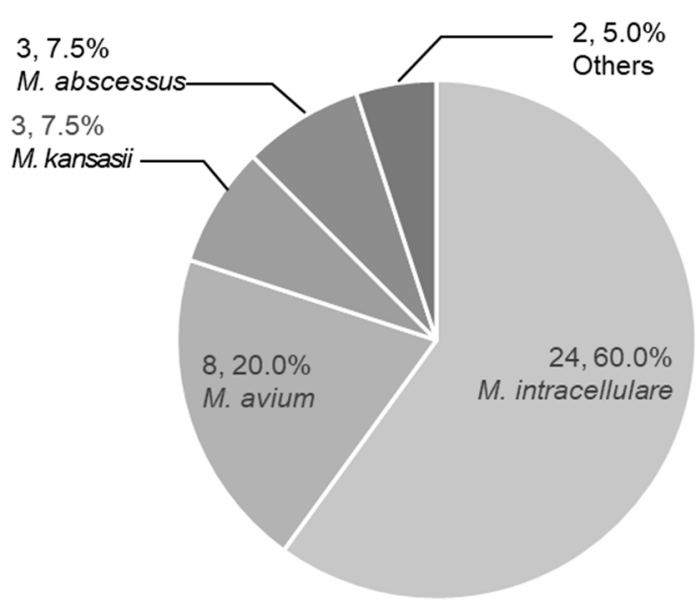

Figure 1. Microbiological findings. Etiology of treated elderly patients. Numbers of patients and percentages are shown. 
Table 1. Baseline characteristics of treated elder patients with pulmonary nontuberculous mycobacterial disease.

\begin{tabular}{lc}
\hline \multicolumn{1}{c}{ Characteristics } & Treated Elder \\
& $\mathbf{n}=40$ \\
\hline Age (years old) & $79.1(0.8)$ \\
Gender (female) & $26(65.0)$ \\
BMI $\left(\mathrm{kg} / \mathrm{m}^{2}\right)$ & $20.0(3.6)$ \\
Patient With Smoking History & $14(41.2)$ \\
Patient With Habit of Alcohol & $8(25.8)$ \\
Comorbidities & $24(60.0)$ \\
Respiratory Disease & $18(45.0)$ \\
Malignancy & $9(22.5)$ \\
Heart Disease & $7(17.5)$ \\
Patient with ADL Disability & $12(30.0)$ \\
Symptoms & \\
Cough & $23(57.5)$ \\
Sputum & $20(50.0)$ \\
Hemoptysis & $10(25.0)$ \\
Dyspnea & $2(5.0)$ \\
Fever & $5(12.5)$ \\
Weight Loss & $6(15.0)$ \\
Radiological Type & \\
Fibrocavitary & $14(35.0)$ \\
Nodular Bronchiectatic & $26(65.0)$ \\
More Than Three Lobes Involved Based on CT & $22(55.0)$ \\
Diagnosed by Bronchofiberscopy & $7(17.5)$ \\
Prescribed Number of Antimycobacterial Drugs & \\
Three or More Drugs & $28(70.0)$ \\
Two Drugs & $3(7.5)$ \\
Single Drug & $9(22.5)$ \\
Use of Standard Regimens * & $24(60.0)$ \\
\hline
\end{tabular}

Abbreviations: $\mathrm{ADL}=$ activities of daily living; $\mathrm{AMK}$ = amikacin; $\mathrm{BMI}=$ body mass index; $\mathrm{CAM}=$ clarithromycin; $\mathrm{CT}$ = computed tomography; $\mathrm{EB}=$ ethambutol; $\mathrm{INH}=$ isoniazid; IPM/CS = imipenem/cilastatin; $\mathrm{RFP}=$ rifampicin . Data are presented as $\mathrm{n}(\%)$ for categorical data or mean values (standard deviation) for numeric data. * Standard regimens were defined as combination of RFP, EB, and CAM for M. avium complex, combination of INH, RFP, and EB for M. kansasii, and combination of AMK, CAM, and IPM/CS for M. abscessus.

\subsection{Continuation of Treatment}

Treatment was continued for 12 months in 22/40 (55\%) patients. Specifically, 15/28 (53.6\%), 1/3 $(33.3 \%)$, and $6 / 9(66.7 \%)$ patients were able to continue regimens with three or more drugs, two drugs, and single drug respectively (Figure 2). Discontinuation due to treatment-related AEs were observed in $8 / 18(44.4 \%)$ patients of all discontinued or changed patients. A comparison of the characteristics between treated elderly patients who changed or discontinued the treatment $(n=18)$ and those who were able to continue their treatment $(n=22)$ is presented in Table 2. Patients who changed or discontinued the treatment comprised a greater proportion of patients with ADL disability (nine in 18, $50.0 \%$ vs. three in $22,13.6 \%, p=0.018$ ) and heart disease ( $\operatorname{six}$ in $18,33.3 \%$ vs. one in $22,4.6 \%, p=0.033$ ). Those six patients with heart disease had to discontinue their treatment either due to treatment related AEs (one in six patients) or decline of general status (five in six patients). Nine patients with ADL disability who changed or discontinued their treatment had to discontinue the treatment for treatment related AEs (six in nine patients), decline of general status (two in nine patients), or death by that was not related to NTM infection (one in nine patients). 


\begin{tabular}{|c|c|c|}
\hline \multicolumn{3}{|c|}{ Total of 40 treated elderly pulmonary NTM patients } \\
\hline $\begin{array}{l}\text { More than three drugs } \\
\qquad(n=28)\end{array}$ & $\begin{array}{l}\text { Two drugs } \\
\qquad(n=3)\end{array}$ & $\begin{array}{l}\text { Single drug } \\
(\mathrm{n}=9)\end{array}$ \\
\hline Continued (n=15) & Continued $(n=1)$ & Continued $(n=6)$ \\
\hline $\begin{array}{l}\text { Changed }(n=2) \\
\text { AEs related to treatment }(n=2)\end{array}$ & $\begin{array}{l}\text { Changed }(n=1) \\
\text { AE related to treatment }(n=1)\end{array}$ & \\
\hline $\begin{array}{l}\text { Discontinued }(n=11) \\
\text { AEs related to treatment }(n=5) \\
\text { AEs unrelated to treatment }(n=5) \\
\text { Not effective }(n=1)\end{array}$ & $\begin{array}{l}\text { Discontinued ( } n=1) \\
\text { AE unrelated to treatment }(n=1)\end{array}$ & $\begin{array}{l}\text { Discontinued }(n=3) \\
\text { AEs unrelated to treatment }(n=2) \\
\text { Not effective }(n=1)\end{array}$ \\
\hline
\end{tabular}

Figure 2. Overview of the number of patients according to the number of antibiotics used in the treatment. Furthermore, the patients were divided to treatment continued, changed, or discontinued. Abbreviations: NTM = nontuberculous mycobacterium; AE: adverse event.

Table 2. Comparison of background characteristics of patients who changed/continued or discontinued the treatment.

\begin{tabular}{|c|c|c|c|}
\hline Characteristics & $\begin{array}{l}\text { Changed or Discontinued } \\
n=18(45.0 \%)\end{array}$ & $\begin{array}{c}\text { Continued } \\
n=22(55.0 \%)\end{array}$ & $p$ \\
\hline Age (years old) & $80.2(4.9)$ & $78.1(4.7)$ & 0.169 \\
\hline Gender (female) & $10(55.6)$ & $16(72.7)$ & 0.257 \\
\hline BMI $\left(\mathrm{kg} / \mathrm{m}^{2}\right)$ & $18.8(2.6)$ & $21.0(4.1)$ & 0.097 \\
\hline Patient With Smoking History & $7(46.7)$ & $7(36.8)$ & 0.563 \\
\hline Patient With Habit of Alcohol & $4(26.7)$ & $4(18.2)$ & 0.916 \\
\hline \multicolumn{4}{|l|}{ Comorbidities } \\
\hline Respiratory Disease & $10(55.6)$ & $8(36.4)$ & 0.225 \\
\hline Malignancy & $5(27.8)$ & $4(18.2)$ & 0.705 \\
\hline Impaired Immunity & $5(27.8)$ & $2(9.1)$ & 0.211 \\
\hline Heart Disease & $6(33.3)$ & $1(4.6)$ & 0.033 \\
\hline Patient With ADL Disability & $9(50.0)$ & $3(13.6)$ & 0.018 \\
\hline \multicolumn{4}{|l|}{ Symptoms } \\
\hline Cough & $10(55.6)$ & $13(59.1)$ & 0.822 \\
\hline Sputum & $9(44.4)$ & $12(54.6)$ & 0.525 \\
\hline Hemoptysis & $3(16.7)$ & $7(31.8)$ & 0.465 \\
\hline Dyspnea & $0(0.0)$ & $2(9.1)$ & 0.492 \\
\hline Fever & $4(22.2)$ & $1(4.6)$ & 0.155 \\
\hline Weight Loss & $3(16.7)$ & $3(13)$. & 1.000 \\
\hline Radiological Type & & & 0.641 \\
\hline Fibrocavitary & $7(38.9)$ & $7(31.8)$ & \\
\hline Nodular Bronchiectatic & $11(61.1)$ & $15(68.2)$ & \\
\hline More Than Three Lobes Involved in CT & $11(61.1)$ & $11(50.0)$ & 0.482 \\
\hline Diagnosed by Bronchofiberscopy & $4(22.2)$ & $3(13.6)$ & 0.680 \\
\hline Prescribed Number of & & & 0.583 \\
\hline Antimycobacterial Drugs & & & 0.383 \\
\hline Three or More Drugs & $13(72.2)$ & $15(68.2)$ & \\
\hline Two Drugs & $2(11.1)$ & $1(4.6)$ & \\
\hline Single Drug & $3(16.7)$ & $6(27.3)$ & \\
\hline Standard Regimens * & $11(61.1)$ & $13(59.1)$ & 0.897 \\
\hline $\begin{array}{l}\text { Adverse Events Associated with } \\
\text { Treatment }\end{array}$ & $11(61.1)$ & $8(36.4)$ & 0.203 \\
\hline
\end{tabular}

Abbreviations: $\mathrm{ADL}=$ activities of daily living; $\mathrm{AMK}=$ amikacin; $\mathrm{BMI}=$ body mass index $\mathrm{CAM}=$ clarithromycin; $\mathrm{CT}=$ computed tomography; $\mathrm{EB}=$ ethambutol; $\mathrm{INH}=$ isoniazid; $\mathrm{IPM} / \mathrm{CS}=$ imipenem/cilastatin; $\mathrm{RFP}=$ rifampicin. Data are presented as $\mathrm{n}(\%)$ for categorical data or mean values (standard deviation) for numeric data. * Standard regimens were defined as combination of RFP, EB, and CAM for M. avium complex, combination of INH, RFP, and EB for M. kansasii, and combination of AMK, CAM, and IPM/CS for M. abscessus. 


\subsection{Adverse Events Associated with Treatment}

A comparison of the characteristics between patients with AEs associated with treatment and those without AE is presented in Table 3. AEs associated with NTM treatment were observed in 19 in $40(47.5 \%)$ patients. The most common AE was gastrointestinal symptoms (13 in 40, 32.5\%) and skin rash (five in 40,12.5\%). When AMK or tobramycin were prescribed, therapeutic drug monitoring (TDM) was performed and all patients who took AMK or tobramycin were in the safe range. Also, electrocardiogram (ECG) was monitored occasionally and prolonged QT interval was not detected in any patients. There were no significant differences in characteristics.

Table 3. Comparison of characteristics of patients based on adverse events associated with treatment.

\begin{tabular}{lccc}
\hline \multicolumn{1}{c}{ Characteristics } & AE & No AE & $p$ \\
\hline Age (years old) & $n \mathbf{1 9}(\mathbf{4 7 . 5 \% )}$ & $\mathbf{n = 2 1 ( 5 2 . 5 \% )}$ & \\
Gender (female) & $77.7(5.0)$ & $80.2(4.5)$ & 0.116 \\
BMI (kg/m $\left.{ }^{2}\right)$ & $13(68.4)$ & $13(61.9)$ & 0.666 \\
Patient With Smoking History & $19.5(2.7)$ & $20.4(4.3)$ & 0.341 \\
Patient With Habit of Alcohol & $5(31.3)$ & $9(50)$ & 0.315 \\
Comorbidities & $3(18.8)$ & $5(33.3)$ & 0.433 \\
Respiratory Disease & & & \\
Malignancy & $8(42.1)$ & $10(47.6)$ & 0.761 \\
Impaired Immunity & $2(10.5)$ & $7(33.3)$ & 0.133 \\
Heart Disease & $4(21.1)$ & $3(14.3)$ & 0.689 \\
Patient With ADL Disability & $4(21.1)$ & $3(14.3)$ & 0.574 \\
Symptoms & $8(42.1)$ & $4(19.1)$ & 0.170 \\
Cough & & & \\
Sputum & $12(63.2)$ & $11(52.4)$ & 0.491 \\
Hemoptysis & $10(52.6)$ & $10(47.6)$ & 0.752 \\
Dyspnea & $6(31.6)$ & $4(19.1)$ & 0.473 \\
Fever & $0(0.0)$ & $2(9.5)$ & 0.489 \\
Weight Loss & $2(10.5)$ & $3(14.3)$ & 0.720 \\
Radiological Type & $3(15.8)$ & $3(14.3)$ & 1.000 \\
Fibrocavitary & & & 0.186 \\
Nodular Bronchiectatic & $9(47.4)$ & $5(23.8)$ & \\
More Than Three Lobes Involved in CT & $10(52.6)$ & $16(76.2)$ & \\
Diagnosed by Bronchofiberscopy & $10(52.6)$ & $12(57.1)$ & 0.775 \\
Prescribed Number of & $3(15.8)$ & $4(19.1)$ & 1.000 \\
Antimycobacterial Drugs & & & 0.258 \\
Three or More Drugs & $15(79.0)$ & $13(61.9)$ & \\
Two Drugs & $2(10.5)$ & $1(4.8)$ & \\
Single Drug & $2(10.5)$ & $11(52.4)$ & 0.349 \\
Standard Regimens * & $13(68.4)$ & & \\
\hline
\end{tabular}

Abbreviations: $\mathrm{ADL}=$ activities of daily living; $\mathrm{AMK}=$ amikacin; $\mathrm{BMI}=$ body mass index; $\mathrm{CAM}=$ clarithromycin; $\mathrm{CT}=$ computed tomography; $\mathrm{EB}=$ ethambutol; $\mathrm{INH}=$ isoniazid; $\mathrm{IPM} / \mathrm{CS}=$ imipenem/cilastatin; $\mathrm{RFP}=$ rifampicin. Data are presented as $\mathrm{n}(\%)$ for categorical data or mean values (standard deviation) for numeric data. * Standard regimens were defined as combination of RFP, EB, and CAM for M. avium complex, combination of INH, RFP, and EB for M. kansasii, and combination of AMK, CAM, and IPM/CS for M. abscessus.

\subsection{Efficacy}

A comparison of the characteristics between patients who failed and those who achieved sputum culture conversion is presented in Table 4 . Sputum culture conversion was achieved in 28/40 (70.0\%) patients. Patients who achieved sputum culture conversion were more on standard regimens than patients who failed sputum culture conversion (21 in $28,75.0 \%$ vs. three in $12,25.0 \% ; p=0.005)$. Also, hemoptysis was less seen in patients who achieved sputum culture conversion (four in $28,14.3 \%$ vs. six in $12,50.0 \% ; p=0.041)$. 
Table 4. Comparison of characteristics between patients who had sputum conversion failed or achieved.

\begin{tabular}{|c|c|c|c|}
\hline Characteristics & $\begin{array}{l}\text { Sputum Culture } \\
\text { Conversion Failed } \\
n=12(30.0 \%)\end{array}$ & $\begin{array}{c}\text { Sputum Culture } \\
\text { Conversion Achieved } \\
n=28(70.0 \%)\end{array}$ & $p$ \\
\hline Age (years old) & $79.4(5.5)$ & $78.9(4.7)$ & 0.756 \\
\hline Gender (female) & $9(75.0)$ & $17(60.7)$ & 0.385 \\
\hline BMI $\left(\mathrm{kg} / \mathrm{m}^{2}\right)$ & $18.3(3.7)$ & $20.7(3.4)$ & 0.212 \\
\hline Patient With Smoking History & $4(40.0)$ & $10(41.7)$ & 1.000 \\
\hline Patient With Habit of Alcohol & $1(11.1)$ & $7(31.8)$ & 0.379 \\
\hline \multicolumn{4}{|l|}{ Comorbidities } \\
\hline Respiratory Disease & $6(50.0)$ & $12(31.8)$ & 0.173 \\
\hline Malignancy & $2(16.7)$ & $7(25.0)$ & 0.697 \\
\hline Impaired Immunity & $1(8.3)$ & $6(21.4)$ & 0.652 \\
\hline Heart Disease & $3(25.0)$ & $4(14.3)$ & 0.410 \\
\hline Patient With ADL Disability & $4(33.3)$ & $8(28.6)$ & 1.000 \\
\hline \multicolumn{4}{|l|}{ Symptoms } \\
\hline Cough & $7(58.3)$ & $16(57.1)$ & 0.944 \\
\hline Sputum & $6(50.0)$ & $14(50.0)$ & 1.000 \\
\hline Hemoptysis & $6(50.0)$ & $4(14.3)$ & 0.041 \\
\hline Dyspnea & $1(8.3)$ & $1(3.6)$ & 0.515 \\
\hline Fever & $1(8.3)$ & $4(14.3)$ & 1.000 \\
\hline Weight Loss & $2(16.7)$ & $4(14.3)$ & 1.000 \\
\hline Radiological Type & & & 0.484 \\
\hline Fibrocavitary & $3(25.0)$ & $11(39.3)$ & \\
\hline Nodular Bronchiectatic & $9(75.0)$ & $17(60.7)$ & \\
\hline More Than Three Lobes Involved in CT & $7(58.3)$ & $15(53.6)$ & 0.077 \\
\hline Diagnosed by Bronchofiberscopy & $0(0.0)$ & $7(25.0)$ & 0.081 \\
\hline Prescribed Number of & & & 0116 \\
\hline Antimycobacterial Drugs & & & 0.116 \\
\hline Three or More Drugs & $6(50.0)$ & $22(78.6)$ & \\
\hline Two Drugs & $2(16.7)$ & $1(3.6)$ & \\
\hline Single Drug & $4(33.3)$ & $5(17.9)$ & \\
\hline Standard Regimens * & $3(25.0)$ & $21(75.0)$ & 0.005 \\
\hline
\end{tabular}

Abbreviations: $\mathrm{ADL}=$ activities of daily living; $\mathrm{AMK}=$ amikacin; $\mathrm{BMI}=$ body mass index; $\mathrm{CAM}=$ clarithromycin; $\mathrm{CT}=$ computed tomography; $\mathrm{EB}=$ ethambutol; $\mathrm{INH}=$ isoniazid; $\mathrm{IPM} / \mathrm{CS}=$ imipenem/cilastatin; $\mathrm{RFP}=$ rifampicin . Data are presented as $\mathrm{n}(\%)$ for categorical data or mean values (standard deviation) for numeric data. * Standard regimens were defined as combination of RFP, EB, and CAM for M. avium complex, combination of INH, RFP, and EB for M. kansasii, and combination of AMK, CAM, and IPM/CS for M. abscessus.

A comparison of the characteristics between patients with and without radiographic improvement is presented in Table 5. Radiographic findings improved in 23 out of 40 (57.5\%) patients. Although patients who had a smoking history appeared more in the improved group (11 in $23,47.8 \%$ vs. three in $17,17.6 \% ; p=0.038$ ), the same group were taking more standard regimens prescribed than patients who had no or worse radiographic changes ( 17 in $23,73.9 \%$ vs. 7 in $17,41.2 \% ; p=0.037$ ). Also, it was suggested that, if standard regimens were prescribed, nodular bronchiectatic radiological type seemed to have better outcome (18 in $23,78.3 \%$ vs. 8 in $17,47.1 \%$ ).

Table 5. Comparison of characteristics between patients who had radiologic findings improved or not.

\begin{tabular}{lccc}
\hline \multicolumn{1}{c}{ Characteristics } & Unchanged or Deteriorated & Improved & $\boldsymbol{p}$ \\
\hline Age (years old) & $78.5(4.8)$ & $79.5(5.0)$ & 0.556 \\
Gender (Female) & $14(82.4)$ & $12(52.2)$ & 0.092 \\
BMI (kg/m ${ }^{2}$ ) & $19.9(4.7)$ & $20.1(2.8)$ & 0.622 \\
Patient With Smoking History & $3(17.6)$ & $11(47.8)$ & 0.038 \\
Patient With Habit of Alcohol & $1(5.9)$ & $7(30.4)$ & 0.095 \\
\hline
\end{tabular}


Table 5. Cont.

\begin{tabular}{|c|c|c|c|}
\hline Characteristics & $\begin{array}{l}\text { Unchanged or Deteriorated } \\
\qquad n=17(42.5 \%)\end{array}$ & $\begin{array}{c}\text { Improved } \\
n=23(57.5 \%)\end{array}$ & $p$ \\
\hline \multicolumn{4}{|l|}{ Comorbidities } \\
\hline Respiratory Disease & $7(41.2)$ & $11(47.8)$ & 0.676 \\
\hline Malignancy & $4(23.5)$ & $5(21.7)$ & 0.893 \\
\hline Impaired Immunity & $3(17.6)$ & $4(17.4)$ & 1.000 \\
\hline Heart Disease & $4(23.5)$ & $3(13.0)$ & 0.432 \\
\hline Patient with ADL Disability & $7(41.2)$ & $5(21.7)$ & 0.296 \\
\hline \multicolumn{4}{|l|}{ Symptoms } \\
\hline Cough & $12(70.6)$ & $11(47.8)$ & 0.202 \\
\hline Sputum & $11(64.7)$ & $9(39.1)$ & 0.200 \\
\hline Hemoptysis & $3(17.6)$ & $7(30.4)$ & 0.471 \\
\hline Dyspnea & $1(5.9)$ & $1(4.4)$ & 1.000 \\
\hline Fever & $2(11.8)$ & $3(13.0)$ & 1.000 \\
\hline Weight Loss & $1(5.9)$ & $5(21.7)$ & 0.216 \\
\hline Radiological Type & & & 0.041 \\
\hline Fibrocavitary & $9(52.9)$ & $5(21.7)$ & \\
\hline Nodular Bronchiectatic & $8(47.1)$ & $18(78.3)$ & \\
\hline More Than Three Lobes Involved in CT & $11(64.7)$ & $11(47.8)$ & 0.289 \\
\hline Diagnosed by Bronchofiberscopy & $2(11.8)$ & $5(21.7)$ & 0.677 \\
\hline Prescribed Number of Antimycobacterial Drugs & & & 0.495 \\
\hline Three or More Drugs & $10(58.8)$ & $18(78.3)$ & \\
\hline Two Drugs & $2(11.8)$ & $1(4.4)$ & \\
\hline Single Drug & $5(29.4)$ & $4(17.4)$ & \\
\hline Standard Regimens* & $7(41.2)$ & $17(73.9)$ & 0.037 \\
\hline
\end{tabular}

Abbreviations: $\mathrm{ADL}=$ activities of daily living; $\mathrm{AMK}=$ amikacin; $\mathrm{BMI}=$ body mass index; $\mathrm{CAM}=$ clarithromycin; $\mathrm{CT}$ = computed tomography; $\mathrm{EB}=$ ethambutol; $\mathrm{INH}=$ isoniazid; IPM/CS = imipenem/cilastatin; RFP = rifampicin . Data are presented as $\mathrm{n}(\%)$ for categorical data or mean values (standard deviation) for numeric data. * Standard regimens were defined as combination of RFP, EB, and CAM for M. avium complex, combination of INH, RFP, and EB for M. kansasii, and combination of AMK, CAM, and IPM/CS for M. abscessus.

\section{Discussion}

This study showed that the continuation of NTM treatment among the elderly was dependent on ADL condition and heart disease comorbidity. Secondly, the prescription of standard regimen was related to achieving sputum culture conversion and better outcome of radiological findings. Importantly, about $30 \%$ of elderly patients were administered with single to two drug regimens which were not recommended by the current guidelines $[9,25,26]$.

The presence of heart disease, such as chronic heart failure, previous myocardial infarction, and arrythmia were related to discontinuation of NTM treatment among our study patients. In a previous study, death due to NTM progression occurred in only $14.6 \%$ of patients, and the main cause of death was non-pulmonary disease (53.8\%) [6]. Along this line, numbers of comorbidities were considered to be an important predictor of death [8]. The main causes of death among NTM patients was heart disease and it was reported to be between $12.5 \%$ and $30 \%[18,21]$. Also, others have reported that heart disease was the main risk of mortality [27]. Although, previous reports have not precisely focused on relationship between treatment discontinuation and patient's comorbidities, our current study showed heart disease was related to discontinuation of the treatment, which was novelty of this study [26]. Also, it seemed to be important to continue the standard regimen since the culture conversion and radiological improvement were achieved more in those group in the current study. This study also confirmed that patients who changed or discontinued the treatment had more comorbidity of heart disease. Therefore, comorbidity of heart disease in elderly patients maybe one of the predictors of treatment discontinuation in elderly NTM patients.

ADL disability was significantly observed in patients who had to change or discontinue the initial treatment in this study. Thus, in addition to cardiac comorbidities, ADL disability may serve as a predictor of treatment discontinuation. The older population tend to have degrees of disability and this study adds ADL evaluation is important when treating elder NTM patients, since 
ADL disability was not discussed in previous NTM studies. The only past study reported related to ADL was the relationship between spine muscles, health-related QOL (HRQL), and prognostic physiological parameters, such as BMI and pulmonary function among NTM infected patients [28]. ADL disability was a risk factor for not only death, but treatment-related AEs when treating pulmonary tuberculosis $[29,30]$. Along with choosing the effective drug regimen, nutrition and physical activity care seems to be important when treating elderly patients, since malnutrition and frailty were highly related to loss of ADL in such group [31-33]. According to previous study, non-drug treatment, such as nutrition therapy or physical rehabilitation, was useful when treating elderly patients with NTM disease [31]. Background characteristics should be carefully evaluated when treating elder population group and ADL should be more cared.

Sputum culture conversion was observed among patients who received a guideline-oriented standard regimen in our current study. Elderly patients have comparable efficacy to past reports with young population [10-23]. Similarly, this study indicated that radiological improvement was also observed among patients who received standard regimen. This observation was consistent with sputum culture conversion for NTM and this is supported by past study [34]. Thus, the efficacy of treatment might be limited when prescribing modified regimens, such as single or two drug regimens. Age might not be an obstacle to prescribe the guideline recommended standard regimens in the elderly patients. Further study is warranted to clarify this certain clinical question.

The use of multiple antibiotic regimens for elderly patients, who is over 70 years old, is not still well studied [10]. Several previous studies reported usefulness of two-drug regimen, but comparison to standard regimen was lacked $[35,36]$. Moreover, there were studies that reported $12.6 \%$ of bronchiectatic MAC pulmonary infections were treated with monotherapy and showed some efficacy [6]. These lines of evidence led physicians to use fewer drugs for pulmonary NTM infection treatment among elderly patients due to absence of sufficient clinical study. However, modified therapies, especially macrolide monotherapy is not recommended [24]. In this study, more than half of the patients were able to take regimens with three or more drugs without any AEs, suggesting a better outcome for those patients.

Given that the present study has methodological limitations related to its retrospective nature and this study was conducted in single center. Although we were able to enroll 181 patients, it may raise the risk of bias due to the small number of participants who were finally analyzed. A multi-center prospective study is warranted to clarify the determination of patients eligible for standard regimens among elderly patients with pulmonary NTM disease. At the very least, our findings provide important factors for treatment continuation, as well as the safety and efficacy of NTM treatment in elderly patients with NTM disease.

\section{Conclusions}

Our current data suggested that age may not be an obstacle for receiving the benefits of the treatment of NTM disease in elderly patients. Furthermore, elderly patients without heart disease and ADL disability may have a better rate of continuing the NTM treatment, suggesting a better outcome for this field in an aging society. Selecting standard regimens and not modifying the NTM treatment is important for the maximum treatment efficacy.

Author Contributions: Conceptualization, Y.U., T.H., and J.T.; methodology, Y.U. and T.H.; validation, Y.U. and T.H.; formal analysis, Y.U. and T.H.; investigation, Y.U., T.H., J.T., H.M., H.S. (Haruhisa Saito), and R.H.; resources, Y.U. and T.H.; data curation, Y.U. and T.H.; writing-original draft preparation, Y.U. and T.H.; writing一review and editing, Y.U., T.H., J.T., K.H., and H.S. (Haruhisa Saito); visualization, Y.U. and T.H.; supervision, Y.U., T.H., and H.S. (Hironori Sagara); project administration Y.U. and T.H.. funding acquisition, Y.U., T.H., and H.S. (Hironori Sagara) All authors have read and agreed to the published version of the manuscript.

Funding: This work was supported in part by JSPS KAKENHI (grant number: 18K10654 to T. Homma).

Acknowledgments: This work was supported in part by JSPS KAKENHI (grant number: 18K10654 to T. Homma). The authors would like to thank Clinical Research Support Center of Asahi General Hospital for the system engineering of data collection. The authors would like to thank Enago (www.enago.jp) for the English language review.

Conflicts of Interest: The authors declare no conflict of interest. 


\section{References}

1. Namkoong, H.; Kurashima, A.; Morimoto, K.; Hoshino, Y.; Hasegawa, N.; Ato, M.; Mitarai, S. Epidemiology of Pulmonary Nontuberculous Mycobacterial Disease, Japan1. Emerg. Infect. Dis. 2016, 22, 1116-1117. [CrossRef] [PubMed]

2. Morimoto, K.; Iwai, K.; Uchimura, K.; Okumura, M.; Yoshiyama, T.; Yoshimori, K.; Ogata, H.; Kurashima, A.; Gemma, A.; Kudoh, S. A Steady Increase in Nontuberculous Mycobacteriosis Mortality and Estimated Prevalence in Japan. Ann. Am. Thorac. Soc. 2014, 11, 1-8. [CrossRef] [PubMed]

3. Izumi, K.; Morimoto, K.; Hasegawa, N.; Uchimura, K.; Kawatsu, L.; Ato, M.; Mitarai, S. Epidemiology of Adults and Children Treated for Nontuberculous Mycobacterial Pulmonary Disease in Japan. Ann. Am. Thorac. Soc. 2018, 16, 341-347. [CrossRef] [PubMed]

4. Morimoto, K.; Hasegawa, N.; Izumi, K.; Namkoong, H.; Uchimura, K.; Yoshiyama, T.; Hoshino, Y.; Kurashima, A.; Sokunaga, J.; Shibuya, S.; et al. A Laboratory-based Analysis of Nontuberculous Mycobacterial Lung Disease in Japan from 2012 to 2013. Ann. Am. Thorac. Soc. 2017, 14, 49-56. [CrossRef] [PubMed]

5. Prevots, D.R.; Marras, T.K. Epidemiology of human pulmonary infection with nontuberculous mycobacteria: A review. Clin. Chest Med. 2014, 36, 13-34. [CrossRef] [PubMed]

6. Gochi, M.; Takayanagi, N.; Kanauchi, T.; Ishiguro, T.; Yanagisawa, T.; Sugita, Y. Retrospective study of the predictors of mortality and radiographic deterioration in 782 patients with nodular/bronchiectatic Mycobacterium avium complex lung disease. BMJ Open 2015, 5, e008058. [CrossRef]

7. Marras, T.K.; Vinnard, C.; Zhang, Q.; Hamilton, K.; Adjemian, J.; Eagle, G.; Zhang, R.; Chou, E.; Olivier, K.N. Relative risk of all-cause mortality in patients with nontuberculous mycobacterial lung disease in a US managed care population. Respir. Med. 2018, 145, 80-88. [CrossRef]

8. $\quad$ Andréjak, C.; Thomsen, V.Ø.; Johansen, I.S.; Riis, A.H.; Benfield, T.; Duhaut, P.; Sørensen, H.T.; Lescure, F.-X.; Thomsen, R.W. Nontuberculous Pulmonary Mycobacteriosis in Denmark. Am. J. Respir. Crit. Care Med. 2010, 181, 514-521. [CrossRef]

9. Haworth, C.S.; Banks, J.; Capstick, T.; Fisher, A.J.; Gorsuch, T.; Laurenson, I.F.; Leitch, A.; Loebinger, M.R.; Milburn, H.J.; Nightingale, M.; et al. British Thoracic Society guidelines for the management of non-tuberculous mycobacterial pulmonary disease (NTM-PD). Thorax 2017, 72, ii1-ii64. [CrossRef]

10. Miwa, S.; Shirai, M.; Toyoshima, M.; Shirai, T.; Yasuda, K.; Yokomura, K.; Yamada, T.; Masuda, M.; Inui, N.; Chida, K.; et al. Efficacy of Clarithromycin and Ethambutol forMycobacterium aviumComplex Pulmonary Disease. A Preliminary Study. Ann. Am. Thorac. Soc. 2014, 11, 23-29. [CrossRef]

11. Jeong, B.-H.; Jeon, K.; Park, H.Y.; Kim, S.-Y.; Lee, K.S.; Huh, H.J.; Ki, C.-S.; Lee, N.Y.; Shin, S.J.; Daley, C.L.; et al. Intermittent Antibiotic Therapy for Nodular BronchiectaticMycobacterium aviumComplex Lung Disease. Am. J. Respir. Crit. Care Med. 2015, 191, 96-103. [CrossRef] [PubMed]

12. Evans, A.J.; Crisp, A.J.; Hubbard, R.B.; Colville, A.; Evans, S.A.; Johnston, I.D. Pulmonary Mycobacterium kansasii infection: Comparison of radiological appearances with pulmonary tuberculosis. Thorax 1996, 51, 1243-1247. [CrossRef] [PubMed]

13. Santin, M.; Dorca, J.; Alcaide, F.; González, L.; Casas, S.; López, M.; Guerra, M.R. Long-term relapses after 12-month treatment for Mycobacterium kansasii lung disease. Eur. Respir. J. 2009, 33, 148-152. [CrossRef] [PubMed]

14. Park, H.K.; Koh, W.-J.; Shim, T.S.; Kwon, O.J. Clinical Characteristics and Treatment Outcomes of Mycobacterium kansasiiLung Disease in Korea. Yonsei Med. J. 2010, 51, 552-556. [CrossRef] [PubMed]

15. Shitrit, D.; Baum, G.L.; Priess, R.; Lavy, A.; Shitrit, A.B.-G.; Raz, M.; Shlomi, D.; Daniele, B.; Kramer, M.R. Pulmonary Mycobacterium kansasii Infection in Israel, 1999-2004. Chest 2006, 129, 771-776. [CrossRef] [PubMed]

16. Kobashi, Y.; Matsushima, T.; Oka, M. A double-blind randomized study of aminoglycoside infusion with combined therapy for pulmonary Mycobacterium avium complex disease. Respir. Med. 2007, 101, 130-138. [CrossRef]

17. Griffith, D.E.; Brown-Elliott, B.A.; Wallace, J.R.J. Thrice-Weekly Clarithromycin-Containing Regimen for Treatment of Mycobacterium kansasii Lung Disease: Results of a Preliminary Study. Clin. Infect. Dis. 2003, 37, 1178-1182. [CrossRef] 
18. Campbell, A. Research First randomised trial of treatments for pulmonary disease caused by M avium intracellulare, M malmoense, and M xenopi in HIV negative patients: Rifampicin, ethambutol and isoniazid versus rifampicin and ethambutol. Thorax 2001, 56, 167-172. [CrossRef]

19. Jenkins, A.; Campbell, P.A.; Banks, I.J.; Gelder, C.M.; Prescott, R.J.; Smith, A.P. Clarithromycin vs ciprofloxacin as adjuncts to rifampicin and ethambutol in treating opportunist mycobacterial lung diseases and an assessment of Mycobacterium vaccae immunotherapy. Thorax 2008, 63, 627-634. [CrossRef]

20. Wallace, R.J.; Brown-Elliott, B.A.; McNulty, S.; Philley, J.V.; Killingley, J.; Wilson, R.W.; York, D.S.; Shepherd, S.; Griffith, D.E. Macrolide/Azalide therapy for nodular/bronchiectatic mycobacterium avium complex lung disease. Chest 2014, 146, 276-282. [CrossRef]

21. Mycobacterium kansasii pulmonary infection: A prospective study of the results of nine months of treatment with rifampicin and ethambutol. Research Committee, British Thoracic Society. Thorax 1994, 49, 442-445. [CrossRef]

22. Sauret, J.; Hernández-Flix, S.; Castro, E.; Hernández, L.; Ausina, V.; Coll, P. Treatment of pulmonary disease caused by Mycobacterium kansasii: Results of 18 vs 12 months' chemotherapy. Tuber. Lung Dis. 1995, 76, 104-108. [CrossRef]

23. Banks, J.; Hunter, A.M.; Campbell, I.A.; Jenkins, P.A.; Smith, A.P. Pulmonary infection with Mycobacterium kansasii in Wales, 1970-1979: Review of treatment and response. Thorax 1983, 38, 271-274. [CrossRef] [PubMed]

24. Griffith, D.E.; Brown-Elliott, B.A.; Langsjoen, B.; Zhang, Y.; Pan, X.; Girard, W.; Nelson, K.; Caccitolo, J.; Alvarez, J.; Shepherd, S.; et al. Clinical and Molecular Analysis of Macrolide Resistance inMycobacterium aviumComplex Lung Disease. Am. J. Respir. Crit. Care Med. 2006, 174, 928-934. [CrossRef]

25. Griffith, D.E.; Aksamit, T.; Brown-Elliott, B.A.; Catanzaro, A.; Daley, C.; Gordin, F.; Holland, S.M.; Horsburgh, R.; Huitt, G.; Iademarco, M.F.; et al. An Official ATS/IDSA Statement: Diagnosis, Treatment, and Prevention of Nontuberculous Mycobacterial Diseases. Am. J. Respir. Crit. Care Med. 2007, 175, 367-416. [CrossRef] [PubMed]

26. Daley, C.L.; Iaccarino, J.M.; Lange, C.; Cambau, E.; Wallace, R.J.; Andrejak, C.; Böttger, E.C.; Brozek, J.; Griffith, D.E.; Guglielmetti, L.; et al. Treatment of nontuberculous mycobacterial pulmonary disease: An official ATS/ERS/ESCMID/IDSA clinical practice guideline. Eur. Respir. J. 2020, 56, 2000535. [CrossRef]

27. Jhun, B.W.; Moon, S.M.; Jeon, K.; Kwon, O.J.; Yoo, H.; Carriere, K.C.; Huh, H.J.; Lee, N.Y.; Shin, S.J.; Daley, C.L.; et al. Prognostic factors associated with long-term mortality in 1445 patients with nontuberculous mycobacterial pulmonary disease: A 15-year follow-up study. Eur. Respir. J. 2019, 55, 1900798. [CrossRef]

28. Asakura, T.; Yamada, Y.; Suzuki, S.; Namkoong, H.; Okamori, S.; Kusumoto, T.; Niijima, Y.; Ozaki, A.; Hashimoto, M.; Yagi, K.; et al. Quantitative assessment of erector spinae muscles in patients with Mycobacterium avium complex lung disease. Respir. Med. 2018, 145, 66-72. [CrossRef]

29. Horita, N.; Miyazawa, N.; Yoshiyama, T.; Kojima, R.; Omori, N.; Kaneko, T.; Ishigatsubo, Y. Poor performance status is a strong predictor for death in patients with smear-positive pulmonary TB admitted to two Japanese hospitals. Trans. R. Soc. Trop. Med. Hyg. 2013, 107, 451-456. [CrossRef]

30. Horita, N.; Miyazawa, N.; Yoshiyama, T.; Tsukahara, T.; Takahashi, R.; Tsukiji, J.; Kato, H.; Kaneko, T.; Ishigatsubo, Y. Decreased activities of daily living is a strong risk factor for liver injury by anti-tuberculosis drugs. Respirology 2013, 18, 474-479. [CrossRef]

31. Sinha, P.; Davis, J.; Saag, L.; Wanke, C.; Salgame, P.; Mesick, J.; Horsburgh, C.R.; Hochberg, N.S. Undernutrition and Tuberculosis: Public Health Implications. J. Infect. Dis. 2018, 219, 1356-1363. [CrossRef] [PubMed]

32. Fujita, K.; Ito, Y.; Oguma, T.; Mio, T.; Niimi, A.; Hirai, T. Association between Mycobacterium avium complex lung disease and serum vitamin D status, antimicrobial peptide levels, and bone mineral density. Medicine 2018, 97, e12463. [CrossRef] [PubMed]

33. Hirai, K.; Tanaka, A.; Homma, T.; Kaneko, K.; Uno, T.; Sato, H.; Manabe, R.; Ohta, S.; Kusumoto, S.; Yamaguchi, F.; et al. Comparison of three frailty models and a sarcopenia model in elderly patients with chronic obstructive pulmonary disease. Geriatr. Gerontol. Int. 2019, 19, 896-901. [CrossRef] [PubMed]

34. Griffith, D.E.; Adjemian, J.; Brown-Elliott, B.A.; Philley, J.V.; Prevots, D.R.; Gaston, C.; Olivier, K.N.; Wallace, R.J. Semiquantitative Culture Analysis during Therapy forMycobacterium aviumComplex Lung Disease. Am. J. Respir. Crit. Care Med. 2015, 192, 754-760. [CrossRef] [PubMed] 
35. Diel, R.; Nienhaus, A.; Ringhausen, F.C.; Richter, E.; Welte, T.; Rabe, K.F.; Loddenkemper, R. Microbiologic Outcome of Interventions Against Mycobacterium avium Complex Pulmonary Disease. Chest 2018, 153, 888-921. [CrossRef]

36. Baldwin, S.L.; Larsen, S.E.; Ordway, D.; Cassell, G.; Coler, R.N. The complexities and challenges of preventing and treating nontuberculous mycobacterial diseases. PLoS Negl. Trop. Dis. 2019, 13, e0007083. [CrossRef]

(C) 2020 by the authors. Licensee MDPI, Basel, Switzerland. This article is an open access article distributed under the terms and conditions of the Creative Commons Attribution (CC BY) license (http://creativecommons.org/licenses/by/4.0/). 